\title{
Distributional profiles of homologous open reading frames among bacterial phyla: implications for vertical and lateral transmission
}

${ }^{1}$ Institute for Molecular Bioscience, The University of Queensland, Brisbane, Qld 4072, Australia

2 Canadian Institute for Advanced Research, Program in Evolutionary Biology

${ }^{3}$ Department of Biology, University of Ottawa, Ottawa, Ontario, Canada K1N 6N5

\author{
Mark A. Ragan ${ }^{1,2}$ and Robert L. Charlebois ${ }^{2,3}+$ \\ Author for correspondence: Mark A. Ragan. Tel: +61 73365 1160. Fax: +61 733654388. \\ e-mail:m.ragan@imb.uq.edu.au
}

If open reading frames (ORFs) have been transmitted primarily by vertical descent, the distributional profile of orthologues of each ORF should be congruent with the organismal tree or a subtree thereof. Distributional patterns not reconciled parsimoniously with tree-like descent and loss are prima facie evidence of lateral gene transfer. Herein, a rigorous criterion for recognizing ORF distributions is described and implemented; it does not require the inference of phylogenetic trees, nor does it assume any specific tree. Because lineage-specific differences in rates of sequence change can also generate unexpected distributional patterns, rate artefacts were controlled for by requiring pairwise matches between ORFs to exceed a rigorous inclusion threshold, but absence of a match was assessed against a more-permissive exclusion threshold. Applying this dual-threshold criterion to cross-domain and cross-phylum distributional patterns for ORFs in 23 bacterial genomes, a relative abundance of ORFs was observed that find a match in exactly seven other bacterial phyla; 94-99\% of these ORFs also find matches among the Archaea and/or Eukarya. In the larger (and some smaller) bacterial genomes, ORFs that find matches in exactly one other bacterial phylum are also relatively abundant, but fewer of these have non-bacterial homologues; most of their matches within the Bacteria are to the Proteobacteria and/or Firmicutes, which cannot be sister lineages to all bacteria. ORFs that are neither distributed universally among the Bacteria, nor necessarily shared with topologically adjacent lineages, are preferentially enriched in large bacterial genomes.

Keywords: lateral gene transfer, horizontal gene transfer, genome evolution, comparative genomics

\section{INTRODUCTION}

Since Darwin (1859), biologists have accepted that organisms have diversified via a tree-like process of

Published online ahead of print on 7 December 2001 as DOI 10.1099/ ijs.0.02026-0.

This paper is an elaboration of a presentation to the Society for General Microbiology Special Symposium 'Genomics: beyond the sequence', Heriot-Watt University, Edinburgh, UK, 26-27 March 2001.

tPresent address: NeuroGadgets Inc., 1551 Riverside Drive, Suite 1506, Ottawa, Ontario, Canada K1G 4B5.

More-detailed versions of Tables 1-3 are available as supplementary material in IJSEM Online (http://ijs.sgmjournals.org/).

Abbreviation: LGT, lateral gene transfer. genealogical descent with modification. This common phylogeny serves as a unifying theme in modern biology, not least in systematics and studies of biodiversity. Although developed originally to explain the diversity of living organisms, the Darwinian paradigm has proven exceptionally powerful in application to the evolution of individual genes and gene families. More recently, it has come to be appreciated that the orderly view of the living world inherent in the Darwinian paradigm is, to some extent, complicated by 'lateral' or 'horizontal' events (Syvanen, 1994), in which genes are transferred from branch to branch in the phylogenetic tree of organisms. It appears wellestablished, for instance, that plastids and mitochondria have arisen from endosymbiotic bacteria (Doug- 
las, 1998; Gray, 1999; Gray et al., 1999; Andersson \& Kurland, 1999) and that plasmids can be spread from one bacterial population to another (Spratt \& Maiden, 1999). In these cases, as in others, the underlying lateral nature of the phenomenon was recognized initially from distributions of phenotypes and was only later confirmed by molecular-phylogenetic analyses.

If lateral gene transfer (LGT) is rare, phylogenetic trees inferred from most individual genes should be topologically congruent. In reality, there is much less congruence than a unitary pattern of descent would suggest, and examples are proliferating of distributions most-parsimoniously explained as resulting from LGT (Brown \& Doolittle, 1997; Doolittle \& Logsdon, 1998; Doolittle, 1999; Jain et al., 1999). With the advent of genomics, attempts have recently been made to estimate the extent of non-tree-like descent in complete microbial genomes. The estimated values are higher than many would have suspected: some $18 \%$ of the open reading frames (ORFs) of Escherichia coli (Lawrence \& Ochman, 1998) and $24 \%$ of the ORFs of Thermotoga maritima (Nelson et al., 1999). If these values are correct and representative, LGT may be an important determinant of genomic diversity in bacteria (Ochman et al., 2000).

Comparison of phylogenetic trees is, in principle, the most rigorous approach for identifying putative LGT (Syvanen, 1994). But inference of trees is subject to manifold biases and artefacts and, for large datasets, can be computationally challenging; thus, in practice, it can be difficult to determine whether non-congruence is real (i.e. an ORF has moved laterally) or only apparent (i.e. an incorrect gene-tree topology has been inferred). We and others have therefore sought alternative approaches that do not require the inference of trees. These methods fall into two major categories: those based on statistical comparison of an ORF primarily vis-à-vis its own genome (Lawrence \& Ochman, 1997, 1998; Karlin et al., 1998a; Lawrence \& Roth, 1999; Mrázek \& Karlin, 1999; Worning et al., 2000) and those involving a statistical comparison of an ORF with similar ORFs in other genomes. Among the latter, comparisons have been based on dinucleotide and oligonucleotide frequencies (Karlin et al., 1998b), best BLAST matches (Koonin et al., 1997; Makarova et al., 1999; Nelson et al., 1999) and distributions of normalized BLASTP similarity scores (Clarke et al., 2002). At least in principle, it is possible by each approach to identify ORFs whose presence in a particular genome is anomalous or not parsimoniously explicable, given the composition of that genome itself (the query genome) and others (target genomes).

The approach we introduce herein falls into the latter category, involving cross-genome statistical comparisons of ORFs. Our motivating idea is that at least some of the LGT between major lineages will distribute ORFs in ways not parsimoniously predicted by Darwinian descent-with-modification and that these unexpected profiles can be identified using a comparison tool such as gapped BLAST (Altschul et al., 1997) applied to conceptually translated protein sequences ('BLASTP'). We assume that there is an organismal, or genomic, tree, but we treat its topology as unknown and do not require that ORFs be distributed accordingly. In this communication, we focus on biological domains (kingdoms) and, within the Bacteria, on phyla. Putative LGT at a finer scale can be examined by application of these methods at the appropriate level, e.g. bacterial orders or below.

Five factors jointly determine the observable distribution of ORFs: vertical (Darwinian) descent, catastrophic (irreversible) loss in specific lineages, absolute and relative rates of sequence change, and lateral transfer. Each bears a specific relationship to the underlying organismal tree, whatever its topology. Vertical descent and catastrophic loss are intrinsically tree-like, yielding symplesiomorphies (shared presences or absences unchanged from the ancestral state) and synapomorphies (shared derived states). Rapid sequence change that occurs at similar rates in all lineages also follows a tree-like pattern, but erodes our ability to resolve the topology of the corresponding gene trees and, in extreme cases, can largely obliterate evidence of homology. Sequence change can be dissimilar in different lineages in ways that are independent of phylogeny, instead reflecting populationlevel phenomena or selection. LGT is (by definition) discordant with the organismal tree, although it could conceivably be more frequent among closely related organisms or within a community.

To identify ORFs that have assumed their current distribution by LGT, it is important to recognize only those pairwise similarities best explained as resulting from discrete, all-or-nothing events inconsistent with vertical descent, i.e. to remove from consideration all rapidly evolving ORFs and as many instances of differential, lineage-specific sequence change as can be recognized. By contrast, others (Aravind et al., 1998; Bruccoleri et al., 1998; Nelson et al., 1999) have attempted to draw conclusions about vertical and lateral transmission of genes based on the best pairwise match, even if it is only slightly better than other matches. To distinguish all-or-nothing matches from those that fall at one end of a continuum, we impose dual thresholds, an inclusion threshold for recognizing a significant pairwise match and an exclusion threshold for accepting the absence of a match (Gaasterland \& Ragan, 1998a, b). As the former is made more stringent (e.g. more-negative BLASTP expectation) and the latter more lenient (e.g. less-negative BLASTP expectation), the patterns of ORF distribution that emerge can be interpreted with increasing confidence as having arisen exclusively by vertical descent, loss and LGT.

\section{METHODS}

Conceptually translated protein sequences corresponding to all ORFs in each of 23 bacterial genomes were 
used to query GenBank, using gapped BLAST version 2.0 (Altschul et al., 1997) and masking regions of low sequence complexity. We queried GenBank (release 117.0), not merely the subset of completely sequenced genomes, to maximize the likelihood of finding matches in each biological domain or phylum. We define as bacterial phyla the second rank of categories recognized by the US National Center for Biotechnology Information (NCBI) (http://www.ncbi.nlm. nih.gov). The exclusion threshold was typically set at an expectation value $e \leqslant 1 \cdot 0 \mathrm{e}-5$ (Gaasterland \& Ragan, 1998a, b) to reduce the number of falsepositive matches. We examined a series of inclusion thresholds from $e \leqslant 1 \cdot 0 \mathrm{e}-8$ to $e \leqslant 1 \cdot 0 \mathrm{e}-300$, but here present data obtained mostly at inclusion thresholds of $e \leqslant 1.0 \mathrm{e}-10$ or $e \leqslant 1 \cdot 0 \mathrm{e}-20$. The latter value, in particular, is sufficiently stringent to require that most or all of each query ORF, not only a conservative domain or region, be matched. As such, we identify these match partners as presumptive homologues. We do not assume (as do e.g. Tatusov et al., 1997) that they are orthologues; elsewhere (G. P. D. Clarke, R. G. Beiko, M. A. Ragan \& R. L. Charlebois, unpublished), we implement a reciprocal best-match criterion to identify ORF pairs that are most likely to be orthologues. For automation of searches, implementation of dual thresholds and taxonomic parsing of GenBank, we used software implemented at http:// www.neurogadgets.com (St Jean \& Charlebois, 1998). Lists of ORFs in each of the 23 bacterial genomes are available at http://www.tigr.org/tdb/mdb/mdb. html and http://www.ncbi.nlm.nih.gov/PMGifs/ Genomes/bact.html

\section{RESULTS AND DISCUSSION}

\section{Distributional profiles among bacterial genomes}

We compared ORFs in 23 completely sequenced bacterial genomes against GenBank, sorted the results taxonomically and then filtered with dual thresholds to find all-or-nothing distributional profiles. As can be seen for the ORFs in Escherichia coli K-12 (Table 1), as the inclusion threshold is made increasingly stringent, fewer ORFs are recognized as having all-or-nothing distributional profiles. Opinion differs on how stringent an inclusion threshold should be used for the recognition of a match. In recognizing clusters of orthologous groups, Tatusov et al. (1997) set the BLAST inclusion threshold at $e \leqslant 1 \cdot 0 \mathrm{e}-2$ (and did not implement an exclusion threshold, although they additionally required a mutuality of best match within each cluster of orthologous groups). Gaasterland \& Ragan (1998a), on the other hand, accepted only those pairwise FASTP expectation scores $\leqslant 1 \cdot 0 \mathrm{e}-5$ so as to minimize the numbers of false positives and, even so, recognized that their weaker matches sometimes targeted paralogues. In this study, the most-permissive thresholds we accept are BLASTP $e \leqslant 1.0 \mathrm{e}-10$ for inclusion and BLASTP $e \leqslant 1 \cdot 0 \mathrm{e}-5$ for exclusion. We recognize that, at these thresholds, some homologues will be overlooked, but believe that they should be brought into the analysis on a case-by-case basis by identification of specific structural features (e.g. sequence motifs), not by a general relaxation of thresholds.

Distributional profiles similar to that presented in Table 1 for Escherichia coli were generated for all 23 bacterial genomes. For clarity of presentation, we find it useful to fix a value for the inclusion threshold (the exclusion threshold was set at $e \leqslant 1 \cdot 0 \mathrm{e}-5$ in almost all our analyses) and to project the three remaining axes (query genome, number of target phyla and number or proportion of ORFs) into two dimensions, connecting points for each genome. This can be done for different subsets of query ORFs: those that also find a match outside the Bacteria at a BLASTP expectation value better than the exclusion threshold, those that find no such match, or all query ORFs regardless of match status outside the Bacteria. Fig. 1(a) shows the proportions of ORFs, irrespective of match status outside the Bacteria, that find a match in exactly one, exactly two and so on bacterial target phyla at respective BLASTP inclusion and exclusion thresholds of $e \leqslant 1 \cdot 0 \mathrm{e}-10$ and $e \leqslant 1 \cdot 0 \mathrm{e}-5$. Very similar patterns are found at a succession of more-stringent inclusion thresholds, until numbers of ORFs in some categories become very small for some genomes.

At BLASTP inclusion $e \leqslant 1 \cdot 0 \mathrm{e}-10$ (Fig. 1), 16 of these genomes appear to be relatively enriched in ORFs that find a match in exactly one other bacterial phylum (the phylum represented by the query ORF itself is not counted, as every ORF necessarily matches itself); we term these '1-matchers'. Twenty of the genomes are relatively enriched in ORFs that find matches in exactly seven phyla other than their own, or "7matchers'. Of the seven genomes that do not show relative enrichment in 1-matchers, four are among the smallest known (Mycoplasma genitalium, 467 ORFs; Ureaplasma urealyticum, 611 ORFs; Chlamydia trachomatis, 877 ORFs; Treponema pallidum, 1031 ORFs). The three bacterial genomes that are not relatively enriched in 7-matchers are among the largest released to date: Escherichia coli (4289 ORFs), Bacillus subtilis (4099 ORFs) and Mycobacterium tuberculosis (3918 ORFs). The two thermophiles, Aquifex aeolicus and Thermotoga maritima, exhibit relatively more matches at three, four, five and six other phyla than does any mesophile.

Few ORFs find matches in more than seven other phyla, because the eight phyla represented by these 23 genomes together accounted for $>99.0 \%$ of all bacterial sequences in GenBank (version 117.0). 7Matchers are thus broadly distributed among bacteria, finding matches in all matchable bacterial phyla. In an earlier version of this analysis, conducted using 16 bacterial genomes representing seven phyla (against GenBank version 114.0), this higher mode was observed at six other target phyla; the subsequent release of the Deinococcus radiodurans genome (the first 
Table 1. Numbers of ORFs in the genome of Escherichia coli K-12 finding matches in exactly 1 to 10 bacterial phyla other than its own (Proteobacteria) at selected BLASTP thresholds

BLASTP inclusion thresholds from $e \leqslant 1 \cdot 0 \mathrm{e}-8$ to $e \leqslant 1 \cdot 0 \mathrm{e}-300$ were used. The exclusion threshold was $e \leqslant 1 \cdot 0 \mathrm{e}-5$. Numbers are shown irrespective of whether these ORFs also find matches in the Archaea and/or Eukarya at BLASTP $e \leqslant 1 \cdot 0 \mathrm{e}-5$ (left) or excluding ORFs that also find matches outside the Bacteria (right). A more-detailed version of this Table is available as supplementary material in IJSEM Online (http://ijs.sgmjournals.org/).

\begin{tabular}{|c|c|c|c|c|c|c|c|c|c|c|c|c|c|c|c|c|c|c|c|c|}
\hline \multirow[t]{2}{*}{ Inclusion BLASTP $e$} & \multicolumn{10}{|c|}{$\begin{array}{l}\text { Number of non-self phyla matched irrespective } \\
\text { of non-bacterial matches }\end{array}$} & \multicolumn{10}{|c|}{$\begin{array}{l}\text { Number of non-self phyla matched } \\
\text { (bacteria-only ORFs) }\end{array}$} \\
\hline & 1 & 2 & 3 & 4 & 5 & 6 & 7 & 8 & 9 & 10 & 1 & 2 & 3 & 4 & 5 & 6 & 7 & 8 & 9 & 10 \\
\hline$\leqslant 1 \cdot 0 \mathrm{e}-8$ & 360 & 293 & 217 & 237 & 200 & 194 & 237 & 98 & 104 & 32 & 185 & 87 & 64 & 25 & 17 & 17 & 15 & 0 & 1 & 0 \\
\hline$\leqslant 1 \cdot 0 \mathrm{e}-10$ & 297 & 246 & 175 & 203 & 176 & 161 & 207 & 79 & 93 & 30 & 160 & 76 & 50 & 22 & 14 & 14 & 12 & 0 & 1 & 0 \\
\hline$\leqslant 1 \cdot 0 \mathrm{e}-15$ & 228 & 193 & 113 & 121 & 133 & 111 & 127 & 52 & 64 & 23 & 122 & 58 & 27 & 13 & 9 & 12 & 9 & 0 & 1 & 0 \\
\hline$\leqslant 1 \cdot 0 \mathrm{e}-20$ & 189 & 150 & 82 & 84 & 95 & 68 & 99 & 36 & 37 & 17 & 98 & 41 & 13 & 9 & 5 & 6 & 5 & 0 & 0 & 0 \\
\hline$\leqslant 1 \cdot 0 \mathrm{e}-25$ & 158 & 122 & 58 & 64 & 78 & 48 & 77 & 29 & 26 & 12 & 77 & 34 & 9 & 5 & 4 & 4 & 4 & 0 & 0 & 0 \\
\hline$\leqslant 1 \cdot 0 \mathrm{e}-30$ & 133 & 88 & 39 & 47 & 66 & 36 & 63 & 23 & 23 & 9 & 70 & 29 & 8 & 3 & 3 & 0 & 3 & 0 & 0 & 0 \\
\hline$\leqslant 1 \cdot 0 \mathrm{e}-40$ & 107 & 65 & 25 & 24 & 41 & 27 & 39 & 17 & 18 & 4 & 53 & 19 & 5 & 1 & 0 & 0 & 2 & 0 & 0 & 0 \\
\hline$\leqslant 1 \cdot 0 \mathrm{e}-50$ & 87 & 48 & 19 & 11 & 34 & 19 & 24 & 12 & 14 & 3 & 45 & 11 & 5 & 0 & 0 & 0 & 1 & 0 & 0 & 0 \\
\hline$\leqslant 1 \cdot 0 \mathrm{e}-60$ & 67 & 30 & 18 & 5 & 23 & 11 & 16 & 10 & 11 & 1 & 32 & 6 & 5 & 0 & 0 & 0 & 0 & 0 & 0 & 0 \\
\hline$\leqslant 1 \cdot 0 \mathrm{e}-80$ & 41 & 16 & 10 & 0 & 8 & 8 & 9 & 7 & 5 & 0 & 18 & 1 & 3 & 0 & 0 & 0 & 0 & 0 & 0 & 0 \\
\hline$\leqslant 1 \cdot 0 \mathrm{e}-100$ & 23 & 12 & 7 & 0 & 4 & 3 & 2 & 4 & 3 & 0 & 12 & 1 & 2 & 0 & 0 & 0 & 0 & 0 & 0 & 0 \\
\hline$\leqslant 1 \cdot 0 \mathrm{e}-150$ & 8 & 4 & 3 & 0 & 0 & 0 & 0 & 1 & 1 & 0 & 3 & 1 & 0 & 0 & 0 & 0 & 0 & 0 & 0 & 0 \\
\hline$\leqslant 1 \cdot 0 \mathrm{e}-300$ & 4 & 1 & 1 & 0 & 0 & 0 & 0 & 1 & 1 & 0 & 1 & 0 & 0 & 0 & 0 & 0 & 0 & 0 & 0 & 0 \\
\hline
\end{tabular}

representative of the Thermus/Deinococcus group) provided the diversity of ORF targets sufficient to increase the higher mode discretely to seven other phyla for each query genome.

Similar distributional profiles are observed over a wide range of inclusion thresholds (alternative sections through the large graphic described above). At very stringent thresholds, however, numbers of ORFs eventually decrease to the point where patterns are obscured (results not shown).

When ORFs that find matches at BLASTP $e \leqslant 1 \cdot 0 \mathrm{e}-5$ outside the Bacteria are excluded from consideration, the signal at $\geqslant 7$ other phyla almost disappears; at $e \leqslant 1 \cdot 0 \mathrm{e}-20$ (Fig. 1b), the decrease is $94-99 \%$ across these 23 genomes (weighted mean $96.7 \%$ ). Thus, ORFs that are broadly distributed within the Bacteria (matching all matchable phyla) overwhelmingly have homologues among the Archaea and/or Eukarya. We hypothesize that most of these ORFs have assumed this multi-domain distribution by descent from an ancestor that predated the divergence of the Bacteria. This does not, however, exclude the possibility (not tested here) that some of these ORFs may subsequently have undergone lateral transfer within individual domains, e.g. within the Bacteria. For each of these 23 genomes, these broadly distributed ORFs are fewer in number (range 71-212, weighted mean 144) than, and do not strongly match the functional profile of, the 256-gene set proposed to be minimal for cellular life (Mushegian \& Koonin, 1996).
Similarly, 74-94\% (weighted mean $90.0 \%$ ) of bacterial ORFs that find a match in exactly three, four, five or six other bacterial phyla also find a match in the Archaea and/or Eukarya (Fig. 1a versus Fig. 1b). We hypothesize that many of these patterns have been generated by catastrophic loss, in individual lineages, of ancient ORFs (e.g. during genomic streamlining: Andersson \& Kurland, 1998; Andersson \& Andersson, 1999). A contribution from promiscuous lateral transfer cannot be excluded, but cannot be parsimoniously assumed in the absence of additional evidence (e.g. from a well-supported phylogenetic tree).

\section{1-Matchers: candidates for LGT?}

In contrast to the 7-matchers discussed above, ORFs that find matches in exactly one other bacterial genome show a much higher frequency of matching only bacterial ORFs. Over these 23 bacterial genomes, $40 \cdot 1 \%$ of the 1 -matchers at BLASTP $\leqslant 1 \cdot 0 \mathrm{e}-20$ have no homologue detectable, at BLASTP $e \leqslant 1 \cdot 0 \mathrm{e}-5$, outside the Bacteria. For the distributional profiles of these ORFs to be parsimoniously explicable as the result of vertical descent on a well-resolved tree, each must match a target in the topologically nearest lineage for which sufficient data are available. Keeping in mind that our approach does not assume any specific tree, four lines of evidence indicate that this is not what is observed for most of these genomes. 

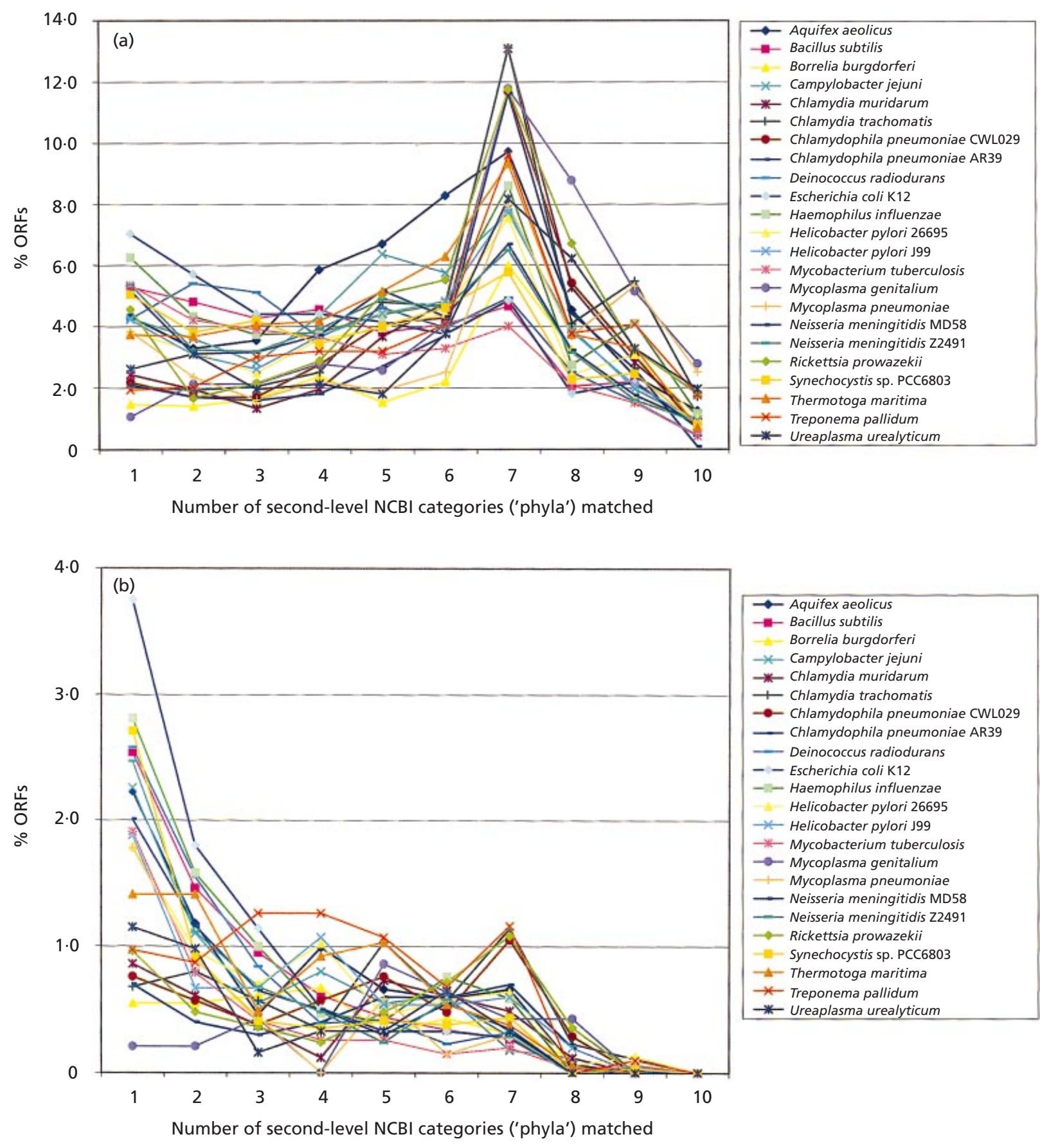

Number of second-level NCBI categories ('phyla') matched

Fig. 1. In each of 23 bacterial genomes, the percentage of ORFs that find matches in exactly one, exactly two, etc. other bacterial phyla (second-level NCBI categories: see Methods) at respective inclusion and exclusion thresholds BLASTP $e \leqslant 1.0 e-10$ and $1.0 e-5$, when ORFs that also find matches at $e \leqslant 1.0 e-5$ in the Archaea and/or Eukarya are (a) included or (b) disqualified from consideration. Thus, ORFs contributing to (a) may be exclusively bacterial or more broadly distributed, whereas those in (b) are exclusively bacterial.

Firstly, the most-matched target phylum is always either the Firmicutes or Proteobacteria (Table 2). In any resolved tree (irrespective of its precise topology), all phyla cannot have the same sister-group; thus, the most-matched target cannot, for most of these query genomes, be topologically adjacent. Were genealogy irrelevant for these bacterial 1-matchers, the expected distribution of matches should be a function only of the relative numbers of potential targets (protein sequences in GenBank) among the other phyla; we term this the 'stochastic model'. Alternatively, if genealogy were an important determinant of how 
Table 2. Phyla targeted by 1 -matchers from 23 bacterial genomes (grouped by phylum) at BLASTP inclusion threshold $e \leqslant 1 \cdot 0 \mathrm{e}-20$ and exclusion threshold $e \leqslant 1 \cdot 0 \mathrm{e}-5$

Phyla are abbreviated as: Aqui, Aquificales; Chly, Chlamydiales; Cyan, Cyanobacteriales; Cytp, Cytophagales; Firm, Firmicutes; GNS, green non-sulfur bacteria; GSB, green sulfur bacteria (includes Cytophaga-Flavobacterium-Bacteroides); Prot,

Proteobacteria; Spir, Spirochaetales; Sym, Symbiobacteria; Ther, Thermotogales; Th/D, Thermus/Deinococcus group (includes Chloroflexus/Deinococcus group); Other, other (inconsistent taxonomic labelling for which aliases were not available at the time of analysis, but since corrected). $\times$, Phylum of the query genome. Numbers shown are for matches in bacterial phyla other than that of the query genome, irrespective of whether these ORFs also find a match outside the Bacteria. A more-detailed version of this Table, showing match distributions for each of the bacterial genomes, is available as supplementary material in IJSEM Online (http://ijs.sgmjournals.org/).

\begin{tabular}{|c|c|c|c|c|c|c|c|c|c|c|c|c|c|c|}
\hline \multirow[t]{2}{*}{ Query phylum } & \multicolumn{13}{|c|}{ Target phylum } & \multirow[t]{2}{*}{ Total } \\
\hline & Aqui & Chly & Cyan & Cytp & Firm & GNS & GSB & Prot & Spir & Sym & Ther & Th/D & Other & \\
\hline Aqui & $x$ & & 1 & & 5 & & & 8 & & & 18 & 1 & & \\
\hline Chly & & $x$ & & & 17 & & & 36 & & & & 4 & & \\
\hline Cyan & 1 & & $x$ & & 24 & & 1 & 32 & & & 6 & 9 & & \\
\hline Firm & 5 & 2 & 21 & 3 & $x$ & & & 210 & & & 15 & 30 & 1 & \\
\hline Prot & 26 & 24 & 70 & 3 & 337 & 1 & 1 & $\times$ & 15 & 1 & 7 & 27 & & \\
\hline Spir & & 2 & 1 & 1 & 5 & & & 10 & $x$ & & 1 & & & \\
\hline Ther & 14 & & 4 & & 12 & & & 9 & 2 & & $x$ & 3 & & \\
\hline Th/D & & 1 & 7 & & 38 & & & 20 & & & 2 & $x$ & 3 & \\
\hline All & 46 & 29 & 104 & 7 & 438 & 1 & 2 & 325 & 17 & 1 & 49 & 74 & 4 & 1097 \\
\hline
\end{tabular}

Table 3. Numbers of matches from 1-matchers as distributed among major target phyla, at BLASTP inclusion threshold $e \leqslant 1 \cdot 0 \mathrm{e}-20$ and exclusion threshold $e \leqslant 1 \cdot 0 \mathrm{e}-5$

See legend to Table 2 for definition of abbreviations. Values expected under the stochastic model (see text) and observed values for query ORFs in the 23 bacterial genomes, grouped by phyla. A more-detailed version of this Table, showing data for each of the bacterial genomes, is available in IJSEM Online (http://ijs.sgmjournals.org/).

\begin{tabular}{|c|c|c|c|c|c|c|c|c|c|c|c|c|c|c|c|c|}
\hline \multirow[t]{3}{*}{ Phylum } & \multicolumn{8}{|c|}{ Requiring match outside bacteria } & \multicolumn{8}{|c|}{ Having no match outside bacteria } \\
\hline & \multicolumn{2}{|c|}{ Firm } & \multicolumn{2}{|c|}{ Prot } & \multicolumn{2}{|c|}{ Cyan } & \multicolumn{2}{|c|}{ All others } & \multicolumn{2}{|c|}{ Firm } & \multicolumn{2}{|c|}{ Prot } & \multicolumn{2}{|c|}{ Cyan } & \multicolumn{2}{|c|}{ All others } \\
\hline & Exp & Obs & Exp & Obs & Exp & Obs & Exp & Obs & Exp & Obs & Exp & Obs & Exp & Obs & $\operatorname{Exp}$ & Obs \\
\hline Aqui & $8 \cdot 5$ & 3 & 11.8 & 5 & $1 \cdot 3$ & 1 & $3 \cdot 4$ & 16 & $2 \cdot 7$ & 2 & $3 \cdot 8$ & 3 & $0 \cdot 4$ & 0 & $1 \cdot 1$ & 3 \\
\hline Chly & $13 \cdot 5$ & 11 & $18 \cdot 7$ & 28 & $2 \cdot 1$ & 0 & $4 \cdot 7$ & 0 & $6 \cdot 2$ & 6 & $8 \cdot 7$ & 8 & $1 \cdot 0$ & 0 & $2 \cdot 1$ & 4 \\
\hline Cyan & $13 \cdot 4$ & 13 & $18 \cdot 7$ & 16 & - & - & 5.9 & 9 & $12 \cdot 3$ & 11 & $17 \cdot 2$ & 16 & - & - & $5 \cdot 5$ & 8 \\
\hline Firm & - & - & $121 \cdot 5$ & 134 & $13 \cdot 9$ & 13 & $38 \cdot 7$ & 29 & - & - & $77 \cdot 5$ & 76 & $8 \cdot 8$ & 8 & $24 \cdot 7$ & 27 \\
\hline Prot & $192 \cdot 8$ & 204 & - & - & $30 \cdot 6$ & 42 & $85 \cdot 5$ & 62 & $127 \cdot 9$ & 133 & - & - & $20 \cdot 3$ & 30 & $56 \cdot 8$ & 42 \\
\hline Spir & 3.5 & 4 & $4 \cdot 8$ & 4 & $0 \cdot 6$ & 0 & $1 \cdot 1$ & 2 & 3.5 & 1 & $4 \cdot 8$ & 6 & $0 \cdot 6$ & 1 & $1 \cdot 1$ & 2 \\
\hline Ther & $10 \cdot 2$ & 6 & $14 \cdot 2$ & 7 & $1 \cdot 6$ & 2 & $4 \cdot 0$ & 15 & $4 \cdot 8$ & 6 & $6 \cdot 6$ & 2 & 0.8 & 2 & $1 \cdot 8$ & 4 \\
\hline Th/D & $11 \cdot 0$ & 18 & $15 \cdot 4$ & 8 & $1 \cdot 8$ & 3 & $3 \cdot 8$ & 3 & $13 \cdot 5$ & 20 & $18 \cdot 7$ & 12 & $2 \cdot 1$ & 4 & $4 \cdot 7$ & 3 \\
\hline All & 253 & 259 & 205 & 202 & 52 & 61 & 147 & 136 & 171 & 179 & 137 & 123 & 34 & 45 & 98 & 93 \\
\hline
\end{tabular}

frequently a potential target phylum is matched, we should observe profiles that deviate from those expected under the stochastic model. In fact, observed numbers of matches are remarkably close to expectations under the stochastic model over all these 23 genomes and in most (but not all) individual phyla and genomes where numbers of matches are sufficient to constitute a fair test (Table 3). The few apparent deviations from expectation are discussed below. The stochastic model as presented here is only approximate, as the populations of both target and query ORFs are non-random due, for example, to the occurrence and differential extent of gene families in the various genomes, the over-representation in GenBank of well-studied genes and many other biases. Even so, we find no compelling evidence, for 1matchers representing most of these eight query phyla, that the profile of their matches among bacterial phyla 
Table 4. Frequencies at which major target phyla are matched by 1-matchers that find matches in the Archaea and/or Eukarya at $e \leqslant 1 \cdot 0 \mathrm{e}-5$ and by Bacteria-only 1 -matchers

See legend to Table 2 for definitions of abbreviations.

\begin{tabular}{|c|c|c|c|c|}
\hline \multirow[t]{2}{*}{ Target phylum } & \multicolumn{2}{|c|}{ Requiring match outside bacteria } & \multicolumn{2}{|c|}{ Having no match outside bacteria } \\
\hline & $1 \cdot 0 e-20$ & $1 \cdot 0 \mathrm{e}-10$ & $1 \cdot 0 e-20$ & $1 \cdot 0 e-10$ \\
\hline Firm & $259 / 657=0.394$ & $382 / 1008=0 \cdot 379$ & $179 / 440=0.407$ & $334 / 873=0 \cdot 383$ \\
\hline Prot & $202 / 657=0 \cdot 307$ & $317 / 1008=0 \cdot 314$ & $123 / 440=0 \cdot 280$ & $227 / 873=0 \cdot 260$ \\
\hline Cyan & $61 / 657=0.093$ & $85 / 1008=0 \cdot 084$ & $45 / 440=0 \cdot 102$ & $83 / 873=0.095$ \\
\hline $\mathrm{Th} / \mathrm{D}$ & $37 / 657=0.056$ & $66 / 1008=0 \cdot 065$ & $36 / 440=0 \cdot 082$ & $85 / 873=0.097$ \\
\hline All others & $98 / 657=0 \cdot 149$ & $158 / 1008=0 \cdot 157$ & $57 / 440=0 \cdot 130$ & $144 / 873=0 \cdot 165$ \\
\hline Firm + Prot & $461 / 657=0.702$ & $699 / 1008=0.693$ & $302 / 440=0.686$ & $561 / 873=0.643$ \\
\hline
\end{tabular}

reflects anything other than numbers of target sequences available.

Secondly, matches among the Firmicutes or Proteobacteria are not found more frequently by Bacteriaonly 1 -matchers than by 1 -matchers that have homologues detectable among Archaea and/or Eukarya at BLASTP $e \leqslant 1 \cdot 0 \mathrm{e}-5$. At inclusion threshold $1 \cdot 0 \mathrm{e}-20$, the frequencies with which the Bacteria-only and the more widely distributed 1-matchers find matches in these two target phyla are $302 / 440=0.69$ and $461 / 657$ $=0.70$, respectively, while, at inclusion threshold $1 \cdot 0 \mathrm{e}-10$, they are respectively $561 / 873=0.64$ and $699 / 1008=0.69$ (Table 4). Thus, the frequency at which the Firmicutes or Proteobacteria are the only bacterial phylum matched is no greater (and indeed slightly less) among Bacteria-only ORFs (which could, in principle, have a specific sister-group relationship with one of these two phyla) than among the morenumerous ORFs that, by virtue of having homologues outside the Bacteria, cannot have an exclusive sistergroup relationship with any single bacterial phylum. (This argument is weakened to the extent that these non-Bacterial homologues might themselves have arisen by cross-domain LGT, but such exceptions would only strengthen the overall case for LGT. The mostbasal branch within the Bacteria constitutes a special case.) Bacteria-only and ancient 1-matchers likewise show similar frequencies of finding matches in the Cyanobacteria (Table 4). Bacteria-only 1-matchers find matches more frequently among the Thermus/Deinococcus group than do putatively ancient 1-matchers, but absolute values are small and, in the absence of a realistic statistical model, it cannot be demonstrated that the increased frequency is significant. Thus, where numbers are adequate to constitute a fair test, we find no evidence for a specific genealogical (vertical) component in the match frequencies of Bacteria-only ORFs vis-à-vis those of ORFs that have non-bacterial homologues.

Thirdly, 1-matchers in the larger bacterial genomes, and in the better-represented phyla, typically find targets among most or all bacterial phyla adequately represented in GenBank (Table 2). Given sufficient numbers of query ORFs, even the topologically mostdistant phyla are therefore matched (although, because no specific tree is assumed, we do not know for each query genome which target phylum is most distant). Finally, analyses below the level of bacterial phylum reveal an even greater diversity of distributional pattern (results not shown).

Although, as described in the first point above, a simple stochastic model explains many aspects of the distributional profiles of these genomes (Table 3), some deviations are apparent. Most notably, ORFs of Aquifex aeolicus find a match in the Thermotogales much more frequently than expected under the stochastic model (for Bacteria-only ORFs at BLASTP inclusion $e \leqslant 1 \cdot 0 \mathrm{e}-20$ and exclusion $e \leqslant 1 \cdot 0 \mathrm{e}-5,0 \cdot 15$ matches expected, 3 observed; for ORFs with nonbacterial matches at $e \leqslant 1 \cdot 0 \mathrm{e}-5,0 \cdot 5$ matches expected, 15 observed). Conversely, ORFs of Thermotoga maritima find a match in the Aquificales more frequently than expected (for Bacteria-only ORFs at the same thresholds, $0 \cdot 2$ matches expected, 3 observed; for ORFs with non-bacterial matches at $e \leqslant 1 \cdot 0 \mathrm{e}-5,0 \cdot 5$ matches expected, 11 observed). The excess of ORFs over expectation is most easily interpreted as a reciprocal sister-group effect between Aquifex aeolicus and Thermotoga maritima. Although the absolute values are small, the excess appears to be somewhat more pronounced among ORFs that also have nonbacterial homologues, as would be expected if the Aquificales and Thermotogales together constitute the most-basal branch among bacteria whose genomes have been sequenced (Aravind et al., 1998, 1999; Kyrpides \& Olsen, 1999; Logsdon \& Faguy, 1999).

Other deviations from expectation apparent from Table 3 include: among the ORFs of Deinococcus radiodurans, an excess of matches against the Firmicutes at the expense of Proteobacteria; among several but not all proteobacteria, an excess of matches against the Firmicutes and Cyanobacteria at the expense of other phyla; and among all firmicutes, a slight but consistent excess of matches against the Proteobacteria at the expense of phyla other than the Cyanobacteria. Nonetheless, given the obvious deficiencies (above) of 
Table 5. Numbers of ORFs in each of 23 bacterial genomes that have presumed homologues (operationally defined as matches at BLASTP inclusion $e \leqslant 1 \cdot 0 \mathrm{e}-10$ and exclusion $e \leqslant 1 \cdot 0 e-5$ ) only in its own bacterial phylum and in Archaea and/or Eukarya

\begin{tabular}{|c|c|c|c|c|}
\hline \multirow[t]{2}{*}{ Query genome } & \multicolumn{4}{|c|}{ Matches } \\
\hline & Archaea & Eukarya & $\begin{array}{c}\text { Archaea or } \\
\text { Eukarya }\end{array}$ & $\begin{array}{c}\text { Archaea and } \\
\text { Eukarya }\end{array}$ \\
\hline Aquifex aeolicus & 28 & 2 & 28 & 2 \\
\hline Bacillus subtilis & 17 & 11 & 26 & 2 \\
\hline Borrelia burgdorferi & 2 & 6 & 8 & 0 \\
\hline Campylobacter jejuni & 7 & 9 & 15 & 1 \\
\hline Chlamydia muridarum & 0 & 10 & 10 & 0 \\
\hline Chlamydia trachomatis & 0 & 9 & 9 & 0 \\
\hline Chlamydophila pneumoniae CWL029 & 0 & 9 & 9 & 0 \\
\hline Chlamydophila pneumoniae AR39 & 0 & 9 & 9 & 0 \\
\hline Deinococcus radiodurans & 14 & 8 & 20 & 2 \\
\hline Escherichia coli & 22 & 25 & 43 & 4 \\
\hline Haemophilus influenzae & 9 & 14 & 22 & 1 \\
\hline Helicobacter pylori 26695 & 4 & 15 & 19 & 0 \\
\hline Helicobacter pylori $\mathrm{J} 99$ & 6 & 12 & 18 & 0 \\
\hline Mycobacterium tuberculosis & 25 & 23 & 47 & 1 \\
\hline Mycoplasma genitalium & 2 & 2 & 4 & 0 \\
\hline Mycoplasma pneumoniae & 0 & 6 & 6 & 0 \\
\hline Neisseria meningitidis MD58 & 5 & 15 & 20 & 0 \\
\hline Neisseria meningitidis Z2491 & 5 & 15 & 20 & 0 \\
\hline Rickettsia prowazekii & 0 & 14 & 14 & 0 \\
\hline Synechocystis sp. PCC6803 & 13 & 96 & 108 & 1 \\
\hline Thermotoga maritima & 39 & 5 & 42 & 2 \\
\hline Treponema pallidum & 2 & 3 & 5 & 0 \\
\hline Ureaplasma urealyticum & 1 & 0 & 1 & 0 \\
\hline
\end{tabular}

the simple stochastic model presented here, we find it remarkable that predictions are so similar to the observed values for the majority of pairwise comparisons.

We have argued, so far, that, because no sister-group signal is apparent for many of the 1-matchers, the distribution of their homologues among bacterial phyla must therefore have involved LGT. This conclusion seems inescapable if the underlying (organismal or genomic) tree is well-resolved. If, however, the underlying tree actually resembles a starburst (i.e. if its internal internodes are much shorter than its terminal branches), there might intrinsically be very little sistergroup signal between phyla. Indeed, in published whole-genome trees (Fitz-Gibbon \& House, 1999; Snel et al., 1999), the basal (phylum-level) branches within each division are typically poorly resolved. However, many gene trees are well-resolved at the level of bacterial phyla (e.g. Brown \& Doolittle, 1997), suggesting that the starburst-like shape of wholegenome trees is itself artefactual. Until the matter is further clarified, an underlying starburst-like tree remains, at least formally, as an alternative explanation for the lack of sister-group signal among 1-matchers.

\section{Profiles and the endosymbiotic origins of organelles}

At the time our analyses were conducted, (nearly) complete sets of genomic ORFs were available only for Saccharomyces cerevisiae and Caenorhabditis elegans among eukaryotes. Given the paucity of genomic sequencing so far among protists, and in view of the importance of using complete sets of genomic ORFs as targets (above), it is premature to attempt a rigorous profile-based analysis of vertical and horizontal patterns of ORF relationship linking bacteria and eukaryotes. Nonetheless, our analyses did reveal an unambiguous signal specifically linking Synechocystis sp. (as a representative of the Cyanobacteria) with eukaryotes. At BLASTP inclusion $e \leqslant 1 \cdot 0 \mathrm{e}-10$ and exclusion $e \leqslant$ $1 \cdot 0 \mathrm{e}-5$, we observe 96 Synechocystis ORFs that find a match in the Eukarya but not in any bacterial phylum other than its own; only one of these ORFs also finds a match at these threshold values in the Archaea (Table 5). Among the 95 ORFs specifically shared between cyanobacteria and eukaryotes are genes encoding components of photosystems I and II, phycobilisome subunits, proteins involved in photosynthesis-related electron transport and haem- and chlorophyll-binding proteins, as well as numerous hypothetical proteins (details at http://www.neurogadgets.com). Thus, our 
profile analysis has recognized and identified the (lateral) origin of plastids from a cyanobacterial precursor.

The corresponding signal for mitochondrial origin is much less easily discernable by this approach. Among proteobacteria, only the $\alpha$-proteobacterium Rickettsia prowazekii finds targets among the Eukarya (but not in the Archaea, nor in the Bacteria other than the Proteobacteria) that are annotated as being involved in mitochondrial energetics (succinate dehydrogenase cytochrome $b_{560}$; ubiquinone biosynthesis; cytochromes $c$ and $c_{1}$; cytochrome- $c$ oxidase). Our analysis alone cannot readily distinguish whether these data indicate the specific $\alpha$-proteobacterial origin of mitochondria or shared retention of ancestral sequence (cf. Ragan \& Gaasterland, 1998).

\section{Profiles in relation to genome size}

The smaller bacterial genomes contain proportionally many more 7-matchers than 1-matchers (Fig. 2a, b). As noted above, most of these 7-matchers have homologues outside the Bacteria (Fig. 2a). Particularly to the extent that these non-bacterial homologues are distributed broadly within the Archaea and/or Eukarya, the default hypothesis must be that these gene families arose before the divergence of the Bacteria from the other organismal domains. Conversely, ORFs with homologues in exactly one other bacterial phylum, particularly those lacking homologues outside the Bacteria detectable even at BLASTP $e \leqslant 1.0 \mathrm{e}-5$ (Fig. 2b), are both relatively and absolutely more abundant in the larger bacterial genomes than in small ones. We have argued (above) that there is little evidence of a genealogical (vertical) component in the distributional profiles of Bacteria-only 1-matchers. Thus, large bacterial genomes have become (or remain) larger, in part, by preferential accumulation (or retention or amplification) of ORFs that are neither ancient, nor preferentially shared with the topologically adjacent lineage - i.e. laterally transferred ORFs.

The distributional patterns explained least parsimoniously by vertical descent are those in which a bacterial ORF finds a match (i) only outside the Bacteria or (ii) in only one bacterial phylum other than its own, regardless of match status outside the Bacteria. (These patterns will be less unparsimonious for the deepest-branching bacterial lineage, but here we do not assume any specific bacterial tree and therefore cannot identify basal phyla.) Among these 23 genomes, $0.4 \%$ (Mycoplasma genitalium) to $4.9 \%$ (Escherichia coli) of ORFs (weighted mean, 3.3\%) exhibit one of these patterns at inclusion threshold $1 \cdot 0 \mathrm{e}-20$ and exclusion threshold $1 \cdot 0 \mathrm{e}-5$. These values rise to $1.9 \%$ (Mycoplasma genitalium) to 8.0\% (Escherichia coli), with weighted mean $5.8 \%$, when the inclusion threshold is relaxed to $1 \cdot 0 \mathrm{e}-10$. For these profiles to have been generated primarily by vertical descent, one must assume unparsimoniously that catastrophic ORF loss
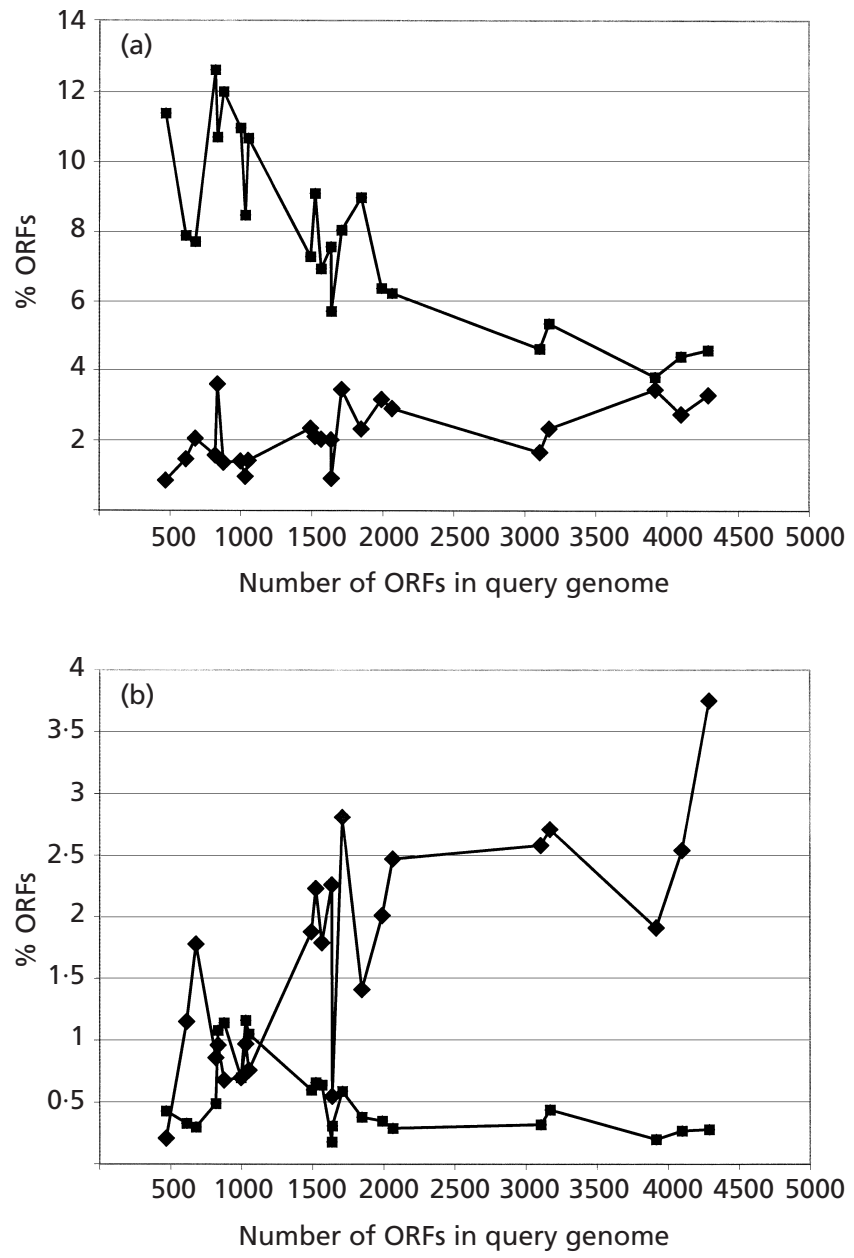

Fig. 2. Relationships between genome size (number of annotated ORFs) and percentage of ORFs finding matches in exactly one $(\bullet)$ or exactly seven $(\boldsymbol{\square})$ other bacterial phyla at respective inclusion and exclusion thresholds BLASTP $e \leqslant 1.0 e-10$ and $1.0 e-5$. (a) ORFs are required to find matches in the Archaea and/or Eukarya; (b) ORFs finding matches outside the Bacteria are disqualified from consideration. Points are connected to aid visual inspection.

and/or extreme increases in the rate of sequence divergence have occurred on multiple occasions across different lineages.

These are probably conservative estimates of the proportions of ORFs in these 23 bacterial genomes that show distributional patterns explained most parsimoniously as having been generated by cross-domain and cross-phylum LGT. If LGT is this frequent, it must also have contributed to the proportions of 2matchers, 3-matchers, etc. Reducing the stringency of our inclusion and exclusion thresholds greatly increases the numbers of ORFs in many categories, including 1-matchers, only some of which can be attributed to false positives. On the other hand, vertical descent and multiple losses may have generated some of the unexpected distributions, even if it is unparsimonious to assume this a priori in the absence of 
specific justification on a case-by-case basis. It is, unfortunately, exactly these 1-matchers for which classical phylogenetic analysis (sequence alignment and inference of trees) will prove most problematic, as other bacterial homologues (if any) will necessarily be difficult to identify and align.

\section{Conclusions}

LGT generates an underlying topological incongruence among different gene trees and between gene and organismal trees. Incongruence sometimes first becomes apparent during the inference of phylogenetic trees. The challenge then is to determine whether the observed incongruence is adequately supported by the data and, if so, whether and how the unexpected result might be explicable in the context of vertical transmission. To do this, one typically obtains sequences of homologues in related organismal lineages and then applies techniques of molecular phylogenetics, e.g. alternative methods of inference, parametric and nonparametric bootstrapping, likelihood-ratio tests or more-sophisticated models of sequence change. If the incongruence is well-supported and cannot be reconciled with vertical transmission, it may be regarded as a prima facie example of LGT. In other cases, however, we first observe an aberrant or unexpected pattern of ORF distribution. Particularly where this involves the sporadic distribution of ORFs among divergent taxa, additional sequencing and phylogenetic inference may not be particularly helpful, and alternative approaches may provide better insight into the history of these ORFs.

Herein, we have employed a dual-threshold criterion to identify the more clear-cut instances where ORF distribution is not explained parsimoniously by vertical transmission. We have shown that ORFs that are widely distributed among the Bacteria overwhelmingly also have homologues outside the Bacteria, i.e. are probably ancient; in the absence of evidence to the contrary (e.g. from well-supported but discordant phylogenetic trees), it may be assumed that many of these ORFs have assumed their present-day distribution by tree-like vertical descent. By contrast, distributional profiles of bacterial ORFs that find a match in only one other bacterial phylum are, with a few exceptions, adequately explained by a simple stochastic model that does not take genealogy (vertical transmission) into account; many of these ORFs may therefore have assumed their present-day distribution by LGT. We also identified bacterial ORFs that find no match partners among other bacterial phyla, but have presumed homologues in Archaea and/or Eukarya. In the case of Synechocystis sp., transmission has been from bacterium to eukaryote during the endosymbiotic origin of chloroplasts.

Heretofore, most attention has attended LGT at the micro-scale (transmission of antibiotic resistance) and at the mega-scale (origins of organelles). The analysis herein suggests that LGT has occurred between many phyla of bacteria. Preliminary analyses show profiles suggestive of LGT at the level of bacterial orders or families as well (M. A. Ragan \& R. L. Charlebois, unpublished), although we await further genomicsequence data to conduct this analysis with satisfactory resolution.

We believe that it is important to have initiated this analysis of distributional profiles using relatively conservative inclusion and exclusion thresholds. As moresophisticated approaches are developed to identify more-divergent homologues, it is likely that estimates of potential LGT will increase. Using the conservative approach outlined above, we find a smaller extent of cross-domain LGT than has previously been estimated (Jain et al., 1999; Nelson et al., 1999; Worning et al., 2000). Nonetheless, our results confirm that bacterial genomes have been shaped, in part, by processes very different from those that Darwin would have imagined.

\section{ACKNOWLEDGEMENTS}

We thank Jeffrey Lawrence for the list of Escherichia coli ORFs and W. Ford Doolittle for advice and critical reading of the manuscript. M.A.R. thanks the Society for General Microbiology for the invitation to participate in SGM-148.

\section{REFERENCES}

Altschul, S. F., Madden, T. L., Schäffer, A. A., Zhang, J., Zhang, Z., Miller, W. \& Lipman, D. J. (1997). Gapped BLAST and PSI-BLAST: a new generation of protein database search programs. Nucleic Acids Res 25, 3389-3402.

Andersson, J. O. \& Andersson, S. G. E. (1999). Insights into the evolutionary process of genome degradation. Curr Opin Genet Dev $\mathbf{9}$, 664-671.

Andersson, S. G. E. \& Kurland, C. G. (1998). Reductive evolution of resident genomes. Trends Microbiol 6, 263-268.

Andersson, S. G. E. \& Kurland, C. G. (1999). Origins of mitochondria and hydrogenosomes. Curr Opin Microbiol 2, 535-541.

Aravind, L., Tatusov, R. L., Wolf, Y. I., Walker, D. R. \& Koonin, E. V. (1998). Evidence for massive gene exchange between archaeal and bacterial hyperthermophiles. Trends Genet 14, 442-444.

Aravind, L., Tatusov, R. L., Wolf, Y. I., Walker, D. R. \& Koonin, E. V. (1999). Reply [to Kyrpides \& Olsen, 1999]. Trends Genet 15, 299-300. Brown, J. R. \& Doolittle, W. F. (1997). Archaea and the prokaryoteto-eukaryote transition. Microbiol Mol Biol Rev 61, 456-502.

Bruccoleri, R. E., Dougherty, T. J. \& Davison, D. B. (1998). Concordance analysis of microbial genomes. Nucleic Acids Res 26, 4482-4486.

Clarke, G. D. P., Beiko, R. G., Ragan, M. A. \& Charlebois, R. L. (2002). Deduced genome trees using a filter to eliminate phylogenetically discordant sequences and a distance matrix based on mean normalized BLASTP scores. J Bacteriol 184 (in press).

Darwin, C. (1859). Origin of Species. London: Murray.

Doolittle, W. F. (1999). Phylogenetic classification and the universal tree. Science 284, 2124-2129.

Doolittle, W. F. \& Logsdon, J. M., Jr (1998). Archaeal genomics: do archaea have a mixed heritage? Curr Biol 8, R209-R211.

Douglas, S. E. (1998). Plastid evolution: origins, diversity, trends. Curr Opin Genet Dev 8, 655-661. 
Fitz-Gibbon, S. T. \& House, C. H. (1999). Whole genome-based phylogenetic analysis of free-living microorganisms. Nucleic Acids Res 27, 4218-4222.

Gaasterland, T. \& Ragan, M. A. (1998a). Constructing multigenome views of whole microbial genomes. Microb Comp Genomics 3, 177-192.

Gaasterland, T. \& Ragan, M. A. (1998b). Microbial genescapes: phyletic and functional patterns of ORF distribution among prokaryotes. Microb Comp Genomics 3, 199-217.

Gray, M. W. (1999). Evolution of organellar genomes. Curr Opin Genet Dev 9, 678-687.

Gray, M. W., Burger, G. \& Lang, B. F. (1999). Mitochondrial evolution. Science 283, 1476-1481.

Jain, R., Rivera, M. C. \& Lake, J. A. (1999). Horizontal gene transfer among genomes: the complexity hypothesis. Proc Natl Acad Sci U S A 96, 3801-3806.

Karlin, S., Mrazek, J. \& Campbell, A. M. (1998a). Codon usages in different gene classes of the Escherichia coli genome. Mol Microbiol 29, 1341-1355.

Karlin, S., Campbell, A. M. \& Mrazek, J. (1998b). Comparative DNA analysis across diverse genomes. Annu Rev Genet 32, 185-225.

Koonin, E. V., Mushegian, A. R., Galperin, M. Y. \& Walker, D. R. (1997). Comparison of archaeal and bacterial genomes: computer analysis of protein sequences predicts novel functions and suggests a chimeric origin for the archaea. Mol Microbiol 25, 619-637.

Kyrpides, N. C. \& Olsen, G. J. (1999). Archaeal and bacterial hyperthermophiles: horizontal gene exchange or common ancestry? Trends Genet 15, 298-299.

Lawrence, J. G. \& Ochman, H. (1997). Amelioration of bacterial genomes: rates of change and exchange. J Mol Evol 44, 383-397.

Lawrence, J. G. \& Ochman, H. (1998). Molecular archaeology of the Escherichia coli genome. Proc Natl Acad Sci U S A 95, 9413-9417.

Lawrence, J. G. \& Roth, J. R. (1999). Genomic flux: genome evolution by gene loss and acquisition. In Organization of the Prokaryotic Genome, pp. 263-289. Edited by R. L. Charlebois. Washington, DC: American Society for Microbiology.
Logsdon, J. M., Jr \& Faguy, D. M. (1999). Thermotoga heats up lateral gene transfer. Curr Biol 9, R747-R751.

Makarova, K. S., Aravind, L., Galperin, M. Y., Grishin, N. V., Tatusov, R. L., Wolf, Y.I. \& Koonin, E. V. (1999). Comparative genomics of the Archaea (Euryarchaeota): evolution of conserved protein families, the stable core, and the variable shell. Genome Res $\mathbf{9}$, 608-628.

Mrázek, J. \& Karlin, S. (1999). Detecting alien genes in bacterial genomes. Ann NY Acad Sci 870, 314-329.

Mushegian, A. R. \& Koonin, E. V. (1996). A minimal gene set for cellular life derived by comparison of complete bacterial genomes. Proc Natl Acad Sci U S A 93, 10268-10273.

Nelson, K. E., Clayton, R. A., Gill, S. R. \& 26 other authors (1999). Evidence for lateral gene transfer between Archaea and Bacteria from genome sequence of Thermotoga maritima. Nature 399, 323-329.

Ochman, H., Lawrence, J. G. \& Groisman, E. A. (2000). Lateral gene transfer and the nature of bacterial innovation. Nature 405, 299-304.

Ragan, M. A. \& Gaasterland, T. (1998). Microbial genescapes: a prokaryotic view of the yeast genome. Microb Comp Genomics 3, 219-235.

Snel, B., Bork, P. \& Huynen, M. A. (1999). Genome phylogeny based on gene content. Nat Genet 21, 108-110.

Spratt, B. G. \& Maiden, M. C. (1999). Bacterial population genetics, evolution and epidemiology. Philos Trans R Soc Lond B Biol Sci 354, 701-710.

St Jean, A. \& Charlebois, R. L. (1998). The NeuroGadgets Inc. bioinformatics web service. http://www.neurogadgets.com

Syvanen, M. (1994). Horizontal gene transfer: evidence and possible consequences. Annu Rev Genet 28, 237-261.

Tatusov, R. L., Koonin, E. V. \& Lipman, D. J. (1997). A genomic perspective on protein families. Science 278, 631-637.

Worning, P., Jensen, L. J., Nelson, K. E., Brunak, S. \& Ussery, D. W. (2000). Structural analysis of DNA sequence: evidence for lateral gene transfer in Thermotoga maritima. Nucleic Acids Res 28, 706-709. 